\title{
A biologically inspired algorithm to deal with filter-overlap in retinal models
}

\author{
Basabdatta Sen Bhattacharya* and Steve Furber
}

\author{
Address: School of Computer Science, University of Manchester, Manchester, M13 9PL, UK \\ Email: Basabdatta Sen Bhattacharya* - basab815@gmail.com \\ * Corresponding author
}

from Eighteenth Annual Computational Neuroscience Meeting: CNS*2009

Berlin, Germany. 18-23 July 2009

Published: 13 July 2009

BMC Neuroscience 2009, I0(SuppI I):PI 26 doi:I0.II86/I47I-2202-I0-SI-PI26

This abstract is available from: http://www.biomedcentral.com/I47I-2202/I0/SI/PI26

(c) 2009 Sen Bhattacharya and Furber; licensee BioMed Central Ltd.

\section{Introduction}

A multi-filter LN retinal model to simulate parallel processing by a population of retinal ganglion cells was proposed in [1] to test rank-order codes [2], a spikelatency based neural code. Dealing with filter-overlap in this model has been an area of concern $[3,4]$. This is because data redundancy induced by over-sampling of a point in space affects the quantity of salient information during rapid information transmission [5]. We propose a Filter-overlap Correction algorithm (FoCal) to deal with this problem of over-sampled data. The algorithm is based on the lateral inhibition technique [6] used by sensory neurons to deal with data redundancy [7], so that only salient information is transmitted through the opticnerve bottleneck for rapid object detection and recognition.

Following the seminal work in [6] on lateral inhibition, mutual inhibition was described quantitatively in [8] as

$$
r_{1}=e_{1}-K_{1,2}\left(r_{2}-r_{1,2}^{0}\right) \quad r_{2}=e_{2}-K_{2,1}\left(r_{1}-r_{2,1}^{0}\right),
$$

where $r$ is the response of a receptor unit, $e$ is the excitation supplied by the external stimulus on the receptor, $K$ is the coefficient of inhibitory influence of one receptor over the other, and $r^{0}$ is the threshold frequency. Let $\Phi_{1}$ and $\Phi_{2}$ be two filters sampling an image at spatial locations $(x, y)$ and $(x+1, y)$ respectively on a digital raster, the respective coefficients of filtering $c_{1}$ and $c_{2}$ representing the spiking latency of the retinal ganglion cells corresponding to the filters. The filter overlap is written as $O_{1,2}=O_{2,1}=$ $\left\langle\Phi_{1}, \Phi_{2}\right\rangle$. Let $c_{1}>c_{2}$. The smaller coefficient is corrected for the effect of this overlap thus

$$
r_{2}=c_{2}-O_{2,1} \cdot c_{1},
$$

where $r_{2}$ simulates the overlap corrected response latency of the ganglion cell corresponding to $\Phi_{2}$. However, we introduce lateral inhibition post-spiking whereas it is prespiking in biology. Thus, we substitute the stimulus strength $e_{2}$ with the corresponding coefficient of filtering $c_{2}$. The threshold frequency is also irrelevant in our case. Further, we implement a winner-take-all mechanism in each iteration of the algorithm: the largest coefficient inhibits all others, the degree of inhibition being proportional to $O$, which corresponds to $K$. This reduces redundancy in the coefficient set and helps prioritise salient information, thus enabling rapid recovery of perceptually important information [4] in rank-order encoded images.

\section{Results and conclusion}

Using a data set of 65 images, we obtain the mean perceptually-important information recovery plot as shown (Figure 1). The error-bars show the standard deviation across the data set. We observe an increase of more than $20 \%$ in the total information recovered. Moreover, the rate of information recovery is much faster, with $80 \%$ recovery using the top $10 \%$ of the coefficients, which is a $30 \%$ increase compared to rank-order encoding without using FoCal. Based on these results, we argue that FoCal pro- 


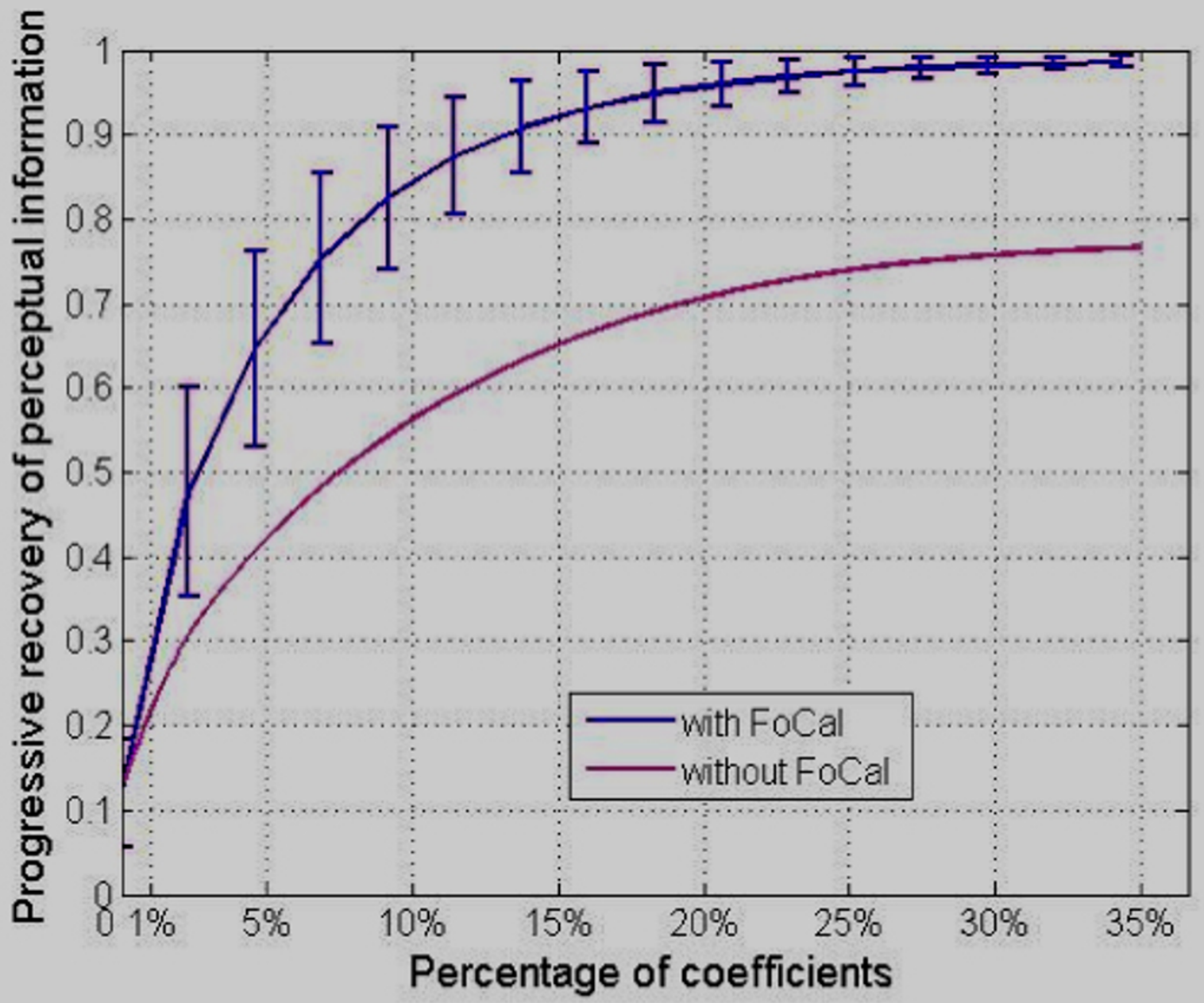

Figure I

vides a general method for coping with non-orthogonal basis functions for current and future biologically inspired visual models.

\section{References}

I. van Rullen $R$, Thorpe $S$ : Rate coding versus temporal order coding: What the retinal ganglion cells tell the visual cortex. Neur Comp 200I, I 3: I 255-I283.

2. Thorpe S, Gautrais J: Rank-order coding. Comp Neur: Trends in Res 1998, 13:113-119.

3. Perrinet L, Samuelides $S$, Thorpe $S$ : Coding static natural images using spiking event times: do neuron cooperate? IEEE Trans NN 2004, 15: I I64-1।75.

4. Sen B: Information recovery from rank-order encoded images. PhD Thesis Uni of Manchester, UK; 2008.

5. Sen B, Furber S: Maximising information recovery from rankorder codes. Proc SPIE DSS 2007, 6570: I- 12

6. Hartline HK: Inhibition of activity of visual receptors by illuminating nearby retinal elements in the limulus eye. Fed Proc 1949:69.

7. Barlow HB: Sensory Coding MIT Press; 1961.

8. Ratliff F: Sensory Coding MIT Press; 1961.
Publish with Biomed Central and every scientist can read your work free of charge

"BioMed Central will be the most significant development for disseminating the results of biomedical research in our lifetime. "

Sir Paul Nurse, Cancer Research UK

Your research papers will be:

- available free of charge to the entire biomedical community

- peer reviewed and published immediately upon acceptance

- cited in PubMed and archived on PubMed Central

- yours - you keep the copyright
BioMedcentral 